\title{
A Study of Anthropometric And Lipid Profile Parameters in Non-Alcoholic Fatty Liver Disease Patients Attending A Tertiary Care Hospitalat Puducherry.
}

\author{
T.M.J.Santhoshakumari ${ }^{1}$, G.Radhika ${ }^{2}, P$. Muraliswaran $^{3}, \mathrm{P}$. Kanagavalli $^{4}$. \\ $\left({ }^{1}\right.$ Post Graduate Student,${ }^{2}$ Professor And Head Of The Department, ${ }^{3}$ Associate Professor, ${ }^{4}$ Professor, \\ Department Of Biochemistry, Sri Venkateshwara Medical College \& Research Centre, Puducherry, India)
}

\begin{abstract}
:
Introduction: Non-alcoholic fatty liver disease (NAFLD )is a common chronic liver disorder sharing the same factors with metabolic syndrome in pathogenesis and insulin resistance. Stress, altered food habits and sedentary life style has led to the development of fatty liver, a marker of metabolic syndrome, leading to cardiovascular disease and Type 2 DM at a younger age. Aim \& objectives : To estimate anthropometric and lipid parameters in NAFLD cases, and controls with normal ultrasonogram (USG).To correlate these parameters with metabolic syndrome. Methodology : A total of 480 patients who were referred for USG from medicine and gynaecology outpatient department were screened. 46 patients with NAFLD and 46 cases with normal USG, of age 18-58 were selected as study and control group respectively. Anthropometric and lipid profile parameters were estimated in both groups. National cholesterol education programme Adult treatment panel III (NCEP-ATPIII) and International Diabetes federation Criteria was used to assess the prevalence of metabolic syndrome. Results: The WC was significantly high in the NAFLD group with a $X^{2}$ value of

25.77 and ' $p$ ' value of 0.0001. The diastolic blood pressure and serum triglycerides had $X^{2}$ value of 17.06 and 6.5 and ' $p$ ' value of 0.00003 and 0.04 respectively. Conclusion: Central obesity and dyslipidemia was significantly higher in the NAFLD group compared to the control group.
\end{abstract}

Keywords: Metabolic syndrome, NAFLD, triglycerides, USG, Waist circumference.

\section{Introduction}

Non-alcoholic fatty liver disease (NAFLD) is defined as the abnormal accumulation of lipids, primarily in the form of triglycerides in individuals with either total abstinence or who do not consume significant amounts of alcohol. ${ }^{1}$ The maximum allowable level of alcohol intake for definition of NAFLD is 2 standard drinks a day ( $140 \mathrm{~g}$ ethanol/week) for men, and one standard drink a day (70 g ethanol/week) for women. ${ }^{2}$ NAFLD is considered as the hepatic representation of metabolic syndrome. Numerous studies have demonstrated that NAFLD is strongly associated with obesity, dyslipidemia, hypertension and insulin resistance, the components of metabolic syndrome. A participant is said to have metabolic syndrome if he/she has two or more of the following criteria as per Modified National Cholesterol Education Programme-Expert Panel-Adult treatment panel III (NCEP-EP-ATP III) criteria. ${ }^{3}$

1. Triglycerides (TG) : >150mg\%.

2. High density lipoprotein (HDL) : $<40 \mathrm{mg} \%$ in male and $<50 \mathrm{mg} \%$ in female.

3. Fasting Blood Sugar : $>100 \mathrm{mg} \%$.

4. Systolic Blood pressure (SBP) $: \geq 130 \mathrm{mmHg}$ and

5. Diastolic blood pressure (DBP) $: \geq 85 \mathrm{~mm} \mathrm{Hg}$

6. Waist circumference (WC) : $>90 \mathrm{~cm}$ in male and $>80 \mathrm{~cm}$ in female.

According to the revised BMI cut-off for Asians ${ }^{4}$ a BMI of 18.6-22.9 is normal, 23-24.9 is overweight and above 25 is considered obese. Urbanization and associated changes, such as sedentary life-style, fat rich diet, and a higher inherited tendency for diabetes mellitus makes Indians more prone to metabolic syndrome or insulin resistance and its manifestations such as NAFLD and NASH.$^{5}$ The prevalence of nonalcoholic fatty liver disease ranges from 9 to $36.9 \%$ of the population in different parts of the world. ${ }^{6}$ The prevalence of NAFLD in Asian countries ranges from 9- $40 \%{ }^{7}$. Indians have increased propensity for visceral fat accumulation which may present from birth. ${ }^{8}$

Prevalence of NAFLD in Indian population was found to be $9-32 \%$ with a higher incidence in over weight and diabetic population according to the epidemiological study conducted between periods of 2004 to 2010. ${ }^{9}$ Hence this study aims at estimating anthropometric and lipid profile parameters in patients, with and without NAFLD so as to assess the risk of cardiovascular morbidity and mortality in future. 


\section{Methodology}

Over a period of 1 year between December 2013-November 2014 about 480 patients from the Gynaecology and Medicine out-patient departments of Sri Venkateswara Medical College and Hospital (SVMCH) who were referred for ultrasonogram were screened after getting written consent. Among them 280 were female and 200 were male of the age group 18-58yrs. From this 46 patients with fatty liver changes in USG were selected as study group after taking detailed history regarding alcohol consumption and drugs. From the remaining 434 patients 46 age matched individuals with normal liver findings in USG were taken as controls. Chronic alcoholism with clinical and imaging features of cirrhosis of liver, use of drugs such as amiodarone, corticosteroids, high dose of oestrogens, tamoxifen or methotrexate and pregnancy were excluded. This study was carried out in collaboration with the Department of Radiology, Sri Venkateshwara Medical College Hospital and Research Centre, Ariyur, Puducherry after obtaining the approval from Institution Ethics Committee.

All patients underwent USG of the abdomen to detect fatty changes in the liver, performed by a single experienced radiologist, for an average of 20 minutes, using a high-resolution B-mode ultrasonography system (SIEMENS-ACUSON x 300 PE, SI no: 330999) having an electric linear transducer mid frequency of 3-5 MHz. Fatty liver was defined as the presence of an ultrasonographic pattern consistent with "bright liver," with evident ultrasonographic contrast between hepatic and renal parenchyma, vessel blurring, and narrowing of the lumen of the hepatic veins in the absence of findings suggestive of chronic liver disease. Electronic weighing machine was used to record the weight to nearest $0.1 \mathrm{Kg}$. Prior to each measurement, the machine was calibrated to zero reading. Height was measured using stadiometer. Body mass index was calculated by the formula BMI $=$ Weight $/$ Height $^{2}\left(\right.$ in $\left.\mathrm{kg} / \mathrm{m}^{2}\right)$. Waist circumference was measured at the midpoint between the lower border of $12^{\text {th }}$ rib and the prominent part of anterior superior iliac spine using a stretch resistant tape. Waist circumference $\geq 90 \mathrm{~cm}$ in male and $\geq 80 \mathrm{~cm}$ in female was considered as central obesity. The systolic and diastolic blood pressure was measured using electronic digital blood pressure monitor, $\mathrm{CH}-432$, CITIZEN Micro Human Tech, Citizen systems Japan Co LTD. Tokyo, Japan after 5 minutes rest.

Fasting blood samples were collected from both groups for estimating lipid profile. The sample was allowed to clot and then centrifuged for 5 minutes at $500 \mathrm{rpm}$ and the plasma used for cholesterol estimation by Trinders method, serum TG by enzymatic colorimetric method and HDL cholesterol by Polyethylene glycol-CHOD-POD method using a well calibrated auto- analyser Chem well. The kits were procured from Erba diagnostics. Serum cholesterol $<200 \mathrm{mg} \%$, TG between 50-150 mg\% and HDL-C values between 40-60 $\mathrm{mg} / \mathrm{dl}$ were considered normal. For the baseline characteristics, continuous variables were summarized by mean (standard deviation) and categorical variables by frequency (percentages $\%$ ). The mean difference and continuous variables were analysed by independent ' $t$ ' test. The association in categorical variables was analysed by chi square test. P value of $<0.05$ was considered significant. The data was analysed using SPSS 17 software.

\section{Results}

As shown in Table $1 \& 2$ in the study group the mean waist circumference was $95.8 \pm 5.24$, BMI $29.53 \pm 5.24$,SBP $129.6 \pm 14.2$, DBP $85.2 \pm 9.9$, TC $190.6 \pm 34.1$, TG $153.7 \pm 78.1$, HDL-C $38.9 \pm 4.4$, and LDL-C $125.9 \pm 33.7$ respectively. In the control group the mean waist circumference was $87.4 \pm 5.8$, BMI $24.74 \pm 2.94$, SBP $124.3 \pm 12.2$, DBP $78.6 \pm 7.1$, TC $162 \pm 31.9$, TG $119.5 \pm 31.6$, HDL $39.7 \pm 4.3$ and LDL $96.9 \pm 32.2$ respectively. There was statistically significant ' $p$ ' value of 0.001 for waist circumference, BMI and DBP between the study group and control group. There was also statistically significant 'p' value between the two groups for TC, TG and LDL-C as shown in table 2 . Table 3 shows statistically significant $\mathrm{X}^{2}$ value of 25.77 \& ' $p$ ' value of 0.0001 for waist circumference in NAFLD group as compared to the control group. DBP shows a $X^{2}$ value of 17.06 and significant 'p' value of 0.011 .

HDL values in male had statistically significant ' $\mathrm{p}$ ' value. As shown in Table 1about $10.9 \%(\mathrm{n}=5)$ were in the normal BMI range of $18-22.9 \mathrm{Kg} / \mathrm{m}^{2}, 8.7 \%(\mathrm{n}=4)$ were in the overweight range (BMI:23-24.9 $\left.\mathrm{Kg} / \mathrm{m}^{2}\right) 32.6 \%(\mathrm{n}=15)$ were in the obese range (BMI: $\left.>25 \mathrm{Kg} / \mathrm{m}^{2}\right)$. Moreover the remaining $47.8 \%(\mathrm{n}=22)$ were in the BMI range above $30 \mathrm{Kg} / \mathrm{m}^{2}$. Thus $89.1 \%$ of NAFLD patients were in overweight and obese range .On the contrary, in the control group $32.6 \%(\mathrm{n}=15)$ were in the normal BMI range, $19.6 \%(\mathrm{n}=9)$ were in the over- weight range, $41.3 \%(\mathrm{n}=19)$ in the obese range and $6.5 \%(\mathrm{n}=3)$ had BMI $>30 \mathrm{Kg} / \mathrm{m}^{2}$. Our study also found a higher waist circumference in $95 \%$ of patients with NAFLD and $25 \%$ in control group. Among the NAFLD group, $34.6 \%(n=16)$ males had waist circumference $>90 \mathrm{~cm}$ and $65.2 \%(\mathrm{n}=30)$ females had waist circumference $>80 \mathrm{~cm}$.

\section{Discussion}

NAFLD has been shown to be an important hepatic correlate of insulin resistance and metabolic syndrome. The BMI, WC, Diastolic and Systolic BP were significantly higher in the NAFLD group compared 
to control group. In the NAFLD group statistically significant increase in TG and decrease in HDL was found. In a study by K. Gokulakrishnan, R.M. Anchana et al at Madras Diabetic Research Foundation and Dr.Mohan's Diabetes Specialities Centre 2009, NAFLD patients had significantly higher BMI, SBP, DBP, TG, and low HDL compared to subjects without NAFLD which is similar to our study.

In our study group about $10.9 \%(\mathrm{n}=5)$ were in the normal BMI range of $18-22.9 \mathrm{Kg} / \mathrm{m}^{2}, 8.7 \%(\mathrm{n}=4)$ were in the overweight range (BMI:23-24.9 Kg/m ${ }^{2}$ ), 32.6\% $(\mathrm{n}=15)$ were in the obese range (BMI: >25 $\mathrm{Kg} / \mathrm{m}^{2}$ ). Moreover the remaining $47.8 \%(\mathrm{n}=22)$ were in the BMI range above $30 \mathrm{Kg} / \mathrm{m} .{ }^{2}$ Thus $89.1 \%$ of NAFLD patients were in overweight and obese range. This confirms the fact that obesity, an important component of MS, would increase with increase in BMI percentiles from underweight to obesity in NAFLD subjects. ${ }^{10}$ This also proves that fatty liver is one of the metabolic surrogate of MS presenting as hepatic insulin resistance.$^{11}$ On the contrary, in the control group $32.6 \%(n=15)$ were in the normal BMI range, $19.6 \%$ $(\mathrm{n}=9)$ were in the over - weight range, $41.3 \%(\mathrm{n}=19)$ in the obese range and $6.5 \%(\mathrm{n}=3)$ had BMI $>30 \mathrm{Kg} / \mathrm{m}^{2}$.

In a study conducted by MES Medical College, ${ }^{12}$ out of 377 NAFLD patients $137(36.3 \%)$ had a body mass index between $21-25 \mathrm{~kg} / \mathrm{m}^{2} .229(60.7 \%)$ patients were in $26-30 \mathrm{~kg} / \mathrm{m}^{2}$ range and the rest 9 $(2.4 \%)$ were having a body mass index more than $30 \mathrm{~kg} / \mathrm{m}^{2}$. Thus $80 \%$ of the NAFLD subjects were in over weight and obese range which is almost similar to our study. In a similar study reported by Chalasani $\mathrm{N}$, Younossi $\mathrm{Z}$ et al about $74 \%$ of patients in NAFLD group were found to be obese. ${ }^{13}$ In a study in rural India, $54 \%$ of NAFLD patients were neither over-weight nor obese which is in contrast to our study. ${ }^{14}$ Probably genetic predisposition, life style, the body build (trunk to leg-length ratio) low muscularity, adaptation to chronic calorie deprivation, and ethnicity ${ }^{15}$ could be a contributing factor forthe difference in this indigenous population.

Most studies report obesity especially central obesity, a common finding in NAFLD as an important risk factor for MS. ${ }^{16}$ Our study also found a higher waist circumference in $95 \%$ of patients with NAFLD and $25 \%$ in control group. Among the NAFLD group, 34.6\% $(\mathrm{n}=16)$ males had waist circumference $>90 \mathrm{~cm}$ and $65.2 \%(\mathrm{n}=30)$ females had waist circumference $>80 \mathrm{~cm}$. These datas are similar to that of Fan et al, ${ }^{17}$ who found the strongest association of central (WC) and overall obesity (BMI) with metabolic syndrome in Asia Pacific region. Central or abdominal obesity, which is more commonly associated with insulin resistance has been observed in 80-90\% of Indian patients with NAFLD. ${ }^{18}$ Similarly Asians have been found to have more intra-abdominal adipose tissue than white Caucasians, in spite of having low waist circumference, ${ }^{19}$ and hence lower cut-offs ( $W C \geq 90 \mathrm{~cm}$ in males and $\geq 80 \mathrm{~cm}$ in females) are recommended for identifying abdominal obesity in Asian Indians.$^{19}$ In a study by Ajay Duseja, ${ }^{20}$ central obesity was found in $96.77 \%$ of subjects which is similar to the findings in our study. Adipose tissue is a dynamic endocrine organ that secretes a number of pro-inflammatory factors like cytokines and adipokines that are involved in the pathogenesis of atherosclerosis, endothelial dysfunction, insulin resistance and vascular remodelling. ${ }^{21}$ The mean systolic blood pressure in the NAFLD group and control group were 129.6 and $123.9 \mathrm{mmHg}$ respectively. The mean diastolic pressure in the NAFLD group and control group were 86.32 and $78.60 \mathrm{mmHg}$. In the NAFLD group $26.08 \%$ of patients $(n=12)$ had higher systolic blood pressure compared to $13.04 \%$ in the control group $(n=6)$. Similarly $56.5 \%$ in the NAFLD group $n=(26)$ and $(15.2 \%)$ in the control group $(n=7)$ had higher diastolic pressure. Hypertension was observed in $10 \%$ to $12 \%$ of patients in a study by Ajay Duseja from New Delhi ${ }^{20}$ which is low compared to our study. Hypertension was found in $45(36.29 \%)$ of respondents in a study by Ajay Duseja ${ }^{22}$ in North India which was high compared to our study. Hypertension, especially systolic hypertension is also an independent NAFLD predictor. ${ }^{23}$ Cotrim et al ${ }^{24}$ confirmed these data by reporting a very high prevalence of $64 \%$ hypertension among NAFLD patients. In a latest study by M.V. Jali, Sanjay Kambar et al ${ }^{25}$ hypertension was present in $85 \%$ of subjects in NAFLD group, which is very high compared to all studies. In contrast, in a study conducted by Kaushal Madan ${ }^{26}$ among Indians and Ajay Dusheja $^{20}$ in another study conducted in New Delhi reported hypertension in 10-12\% of patients in NAFLD group.

Dyslipidemia is known to be a risk factor for NAFLD in most Asia Pacific countries (Amarapurkaret $a l, 2007)$. In our study, in the NAFLD group $26.08 \%(\mathrm{n}=12)$ and in the control group $13.03 \%(\mathrm{n}=6)$ had total cholesterol > 200mg\% and LDL cholesterol calculated using Friedwalds formula ${ }^{27}$ was found to be > $100 \mathrm{mg} \%$ in $21.7 \%(\mathrm{n}=10)$ of the NAFLD group. Triglyceride levels were significantly higher among patients with NAFLD. About 26\% $(\mathrm{n}=12)$ subjects in the NAFLD group had higher TG while it was $6.5 \%(\mathrm{n}=3)$ in the control group. The HDL cholesterol $<40 \mathrm{mg} \%$ was observed in $75 \%(\mathrm{n}=12)$ and in $50 \%(\mathrm{n}=10)$ male subjects in the NAFLD group and control group respectively. It was $<50 \mathrm{mg} \%$ in $100 \%(\mathrm{n}=30)$ female subjects in the NAFLD group and $69 \%(n=18)$ in the control group. This may be attributed to the increased intake of junk foods rich in trans- fatty acids which may have a role in increasing LDL cholesterol and lowering HDL cholesterol. Contrast to our study, in a study by Ajay Duseja, ${ }^{23}$ low HDL cholesterol and high triglyceride level were detected in $38.71 \%$ and $66.13 \%$ of NAFLD patients respectively. In a latest study by M.V.Jali, ${ }^{25}$ dyslipidemia has been reported in high percentage of patients with NAFLD. In a study done at MES Medical 
college, Kerala by Sreenag M, Jimnaz PA et.al ${ }^{21}$ TG was raised in $72.4 \%$ and HDL was low in $77.5 \%$ of patients with NAFLD. Unhealthy diet and lack of exercise could be the reason for high incidence of dyslipidemia. The study by Luxmi et al ${ }^{28}$ also reported raised serum triglyceride level in patients with fatty liver and the same findings was also observed in our study.

Table no.1 The clinical and anthropometric parameters between the two groups.

\begin{tabular}{|c|c|c|c|c|}
\hline & NAFLD & Control & & \\
\hline Parameters & group & group & ' $t$ ' & 'p' \\
\hline & $($ mean \pm SD $)$ & $($ mean \pm SD $)$ & & \\
\hline Age & $41.26 \pm 8.36$ & $38.5 \pm 9.19$ & 1.516 & 0.133 \\
\hline $\mathrm{W} \mathrm{C}$ in $\mathrm{cm}$ & $95.83 \pm 7.4$ & $87.4 \pm 5.8$ & 6.043 & $0.001^{*}$ \\
\hline $\mathrm{BMI}-\mathrm{Kg} / \mathrm{m}^{2}$ & $29.53 \pm 5.24$ & $24.74 \pm 2.94$ & 5.397 & $0.001^{*}$ \\
\hline Systolic BP in & $129.6 \pm 14.2$ & $124.3 \pm 12.2$ & 1.941 & 0.055 \\
\hline \multicolumn{5}{|l|}{ DiastolicBP } \\
\hline $\mathrm{mmHg}$ & $85.2 \pm 9.9$ & $78.60 \pm 7.1$ & 3.697 & $0.001^{*}$ \\
\hline
\end{tabular}

Table no.2 Comparison of Biochemical parameters of NAFLD group and control group.

\begin{tabular}{|c|c|c|c|c|c|c|}
\hline & \multicolumn{2}{|c|}{ NAFLD } & \multicolumn{2}{|c|}{ Control } & \multirow[b]{2}{*}{ ' $t$ ' } & \multirow[b]{2}{*}{ 'p' } \\
\hline Parameters & group & & group & & & \\
\hline in $\mathrm{mg} / \mathrm{dl}$ & \multicolumn{2}{|c|}{$($ mean \pm SD $)$} & \multicolumn{2}{|c|}{$($ mean \pm SD $)$} & & \\
\hline \multicolumn{7}{|l|}{ Total } \\
\hline Cholesterol & 190.6 & \pm 34.1 & 162.0 & \pm 31.9 & 4.146 & $0.001^{*}$ \\
\hline TG & 153.7 & \pm 78.1 & 119.5 & \pm 31.6 & 2.755 & $0.007^{*}$ \\
\hline HDL & 38.9 & \pm 4.4 & 39.7 & \pm 4.3 & 0.883 & 0.379 \\
\hline LDL & 125.9 & \pm 33.7 & \multicolumn{2}{|c|}{$96.9 \pm 32.2$} & 4.227 & $0.001^{*}$ \\
\hline
\end{tabular}

Table no. 3 Components of metabolic syndrome

\begin{tabular}{|c|c|c|c|c|c|}
\hline \multirow{2}{*}{\multicolumn{2}{|c|}{$\begin{array}{c}\text { Components of } \\
\text { Metabolic }\end{array}$}} & NAFLD & Control & \multirow[t]{2}{*}{$\mathrm{X}^{2}$} & \multirow[t]{2}{*}{ 'p' } \\
\hline & & \multirow[t]{2}{*}{ Group } & \multirow[t]{2}{*}{ Group } & & \\
\hline & & & & test & value \\
\hline \multicolumn{2}{|c|}{ syndrome } & $\mathbf{N}(\%)$ & $\mathbf{N}(\%)$ & & \\
\hline \multicolumn{2}{|l|}{ BMI-Kg/m ${ }^{2}$} & $31(67 \%)$ & $31(67 \%)$ & NA & NA \\
\hline & Male & $16(100 \%)$ & $3(15 \%)$ & 25.77 & $0.0001^{*}$ \\
\hline \multirow[t]{3}{*}{ WC } & $>90 \mathrm{~cm}$ & & & & \\
\hline & Female & $30(100 \%)$ & $8(30.1 \%)$ & 30.61 & $.0000001^{*}$ \\
\hline & $>80 \mathrm{~cm}$ & & & & \\
\hline S BP & & $12(26 \%)$ & $6(13 \%)$ & 2.486 & 0.1149 \\
\hline \multicolumn{2}{|c|}{$\geq 135 \mathrm{mmHg}$} & & & & \\
\hline D BP & & $26(56 \%)$ & $7(15 \%)$ & 1706 & $000003^{*}$ \\
\hline & & & & & \\
\hline \multicolumn{2}{|c|}{$\geq 85 \mathrm{mmHg}$} & & & & \\
\hline & TG & & & & \\
\hline & & $12(26 \%)$ & $3(6.5 \%)$ & 6.452 & $0.011^{*}$ \\
\hline \multicolumn{2}{|l|}{$>150 \mathrm{mg} \%$} & & & & \\
\hline & & & & & \\
\hline & Male & & & & \\
\hline & & $12(75 \%)$ & $10(50 \%)$ & 5.3 & $0.021^{*}$ \\
\hline HDL & $<40$ & & & & \\
\hline $\mathrm{mg} \%$ & Female & $30(100 \%)$ & $18(69 \%)$ & 3.125 & 0.077 \\
\hline & $<50$ & & & & \\
\hline
\end{tabular}

\section{Conclusion}

A definite association was observed between the anthropometric, clinical and biochemical parameters in NAFLD patients and the components of metabolic syndrome. Prevention is better than cure. Hence, USG evidence of fatty liver should be taken seriously as a predictor of metabolic syndrome as it is atherogenic and predisposes to diabetes, hypertension, dyslipidemia and has a strong potential for development of coronary vascular disease and T2DM at a younger age. The main limitation of our study is the sample size. 


\section{References}

[1]. Longo, Fauci, Kasperet al, Harrisons Principles of Internal Medicine 18th Edition, Vol : 2 2012; McGraw Hill companies, page: 2604-606.

[2]. Hazlehurst JM, Tomlinson JW (2013) Non-alcoholic fatty liver disease in common endocrine disorders. Eur, J Endocrinol 169: R 27-37.

[3]. Gupta A, Gupta R, Sarna M, Rastogi S, Gupta VP, Kothari K. Prevalence of diabetes, impaired fasting glucose and insulin resistance syndrome in an urban Indian population. Diabetes Res Clin Pract 2003; 61 : 69-76.

[4]. Executive summary of the third report of the National Cholesterol Education Program Expert Panel (Adult Treatment Panel III) on detection, evaluation, and treatment of high blood pressure JAMA 172, 2001; $285: 2486-97$.

[5]. Amarapurkar D, Kamani P, Patel N, Gupte P, Kumar P, Agal S et al, Prevalence of non-alcoholic fatty liver disease: population based study. Ann Hepatol 2007;6:161-3.

[6]. Shen L, Fan JG, Shao Y, Zeng MD, Wang JR, Luo GH et al. (2003). "Prevalence of non-alcoholic fatty liver among administrative officers in Shanghai: an epidemiological survey". World J Gastroenterol 9: 1106-10.

[7]. Bellentani S, Bedogni G et al. Epidemiology of non- alcoholic fatty liver disease. Dig Dis 2010;28:155-61.

[8]. Amarapurkar DN, Hashimoto E, Goh KL et al. How common is non-alcoholic fatty liver disease in the Asia- Pacific region and are there local differences? J Gastroenterol, Hepatol 2007; 22: 788-93.

[9]. Duseja A., Non-alcoholic fatty liver disease in India - a lot done, yet more required. Indian J Gastroenterol 2010;29:217-25.

[10]. Misra A, Chowbey P, Makkar BM et al. Consensus statement for diagnosis of obesity, abdominal obesity and the metabolic syndrome for Asian Indians and recommendations for physical activity, medical and surgical management. J Assoc Physicians India. 2009:57:163-70.

[11]. Hsiehet al, 2000; Angulo, 2002; Fan et al, 2005a; Lee et al, 2006; Cave et al, 2007).

[12]. Fan JG, Saibara T, Chitturi S, Kim BI, Sung JJ, Chutaputti A, Asia Pacific Working Party at NAFLD. What are the risk factors and settings for non-alcoholic fatty liver disease in Asia-Pacific?.J. gastero enteral Hepatol 2007; 22 : 794-800.

[13]. Amarapurkar DN, Hashimoto E, Lesmana LA, Sollano JD, Chen PJ, Goh KL. Asia-Pacific Working Party on NAFLD. How common is non-alcoholic fatty liver disease in the Asia-Pacific region and are there local differences? J Gastro enterol Hepatol 2007; 22:788-793.

[14]. Duseja A, Das A, Das R et al. Clinico-pathological profile of Indian patients with non-alcoholic fatty liver disease is different from that in the West. Dig Dis Sci. 2007;52:2368

[15]. Nasreddine L, Naja F, Tabet M et al Obesity is associated with insulin resistance and components of the metabolic syndrome in Lebanese adolescents. Annals of Human Biology, March - April2012; 39(2): 122-128

[16]. Cotrim HP, Parise ER, Oliveira CP et al Non-alcoholic fatty liver disease in Brazil. Clinical and histological profile. Ann Hepatol 2011;10:33-37.

[17]. M.V.Jali, Sanjay Kambar, Prevalence of NAFLD among T2DM patients a cross sectional hospital based study. Al Ameen $\mathbf{J}$ Med Sci; Volume 8, N0:1,2015.

[18]. Madan K, Batra Y, Gupta SD et al. Non-alcoholic fatty liver disease may not be a severe disease at Presentation among Asian Indians. World J Gastro enterol. 2006;12:3400-5.

[19]. Bajaj S, Nigam P, Luthra A et al. A case-control study on insulin resistance, metabolic co-variates and prediction score in nonalcoholic fatty liver disease. Indian J Med Res. 2009;129:285-92.

[20]. Whye Liun Cheah, Ping Yein Lee et al Prevalence of USG diagnosed NAFLD and its association with biochemical and anthropometric measures. Vol: South East Asian journal of Tropical Medicine. Public Health Vol:44 No;2 March 2013.

[21]. Prashanth M, Ganesh HK. etal Prevalence of NAFLD in patients with T2DM. J Assoc Physicians India. 2009; 57:205-10.

[22]. Sreenag M, Jimnaz PA, Abdul Jaleel etal Prevalence of MS among patients with NAFLD in MES Medical College.Annals MedicusVol:1.Issue 3.July 2014

[23]. Luxmi S, Sattar RA, Ara J. Association of non-alcoholic fatty liver with type 2 diabetes mellitus. JLUMHS 2008;188-193.

[24]. Strauss RS, Barlow SE, Dietz WH (2000) Prevalence of abnormal serum aminotransferase values in overweight and obese adolescents. J Pediatr 136:727-733.

[25]. Laiq hussain et al Pakistan J Med Science October-December 2009 (part 1) vol 25 NO; $5: 817-820$ )

[26]. Roli Agarwal, Sunita Mishra.et al. NAFLD and MS. Indian J. Prev. Soc. Med. Vol.42 No.3, 2011.

[27]. Fan JG, Li F, Cai XB, Peng YD, Ao QH, Gao Y (2007) Effects of NAFLD on the development of metabolic disorders J Gastro enterol Hepatol 22: 1086-1091.

[28]. Rector RS, Thyfault JP, Wei Y, Ibdah JA (2008) Non-alcoholic fatty liver disease and the metabolic syndrome: an update. World J Gastro enterol 14: 185-192. 